\title{
Effets résiduel et d'apport continu de gypse sur les propriétés chimiques d'un sol alcalinisé et sur les performances du maïs (Zea mays L.)
}

Nongma Zongo, Juliane Dao, Jean Pascal Désiré Lompo, Delwendé Innocent Kiba, Zacharia Gnankambary, Papaoba Michel Sedogo \& Hassan Bismarck Nacro

Nongma Zongo : DEA, Ingénieur de recherches, Burkinabè, Département Gestion des Ressources Naturelles et Systèmes de Production, Centre National de la Recherche Scientifique et Technologique, Institut de l'Environnement et de Recherches Agricoles (INERA), BP 476 Ouagadougou 01, Burkina Faso, étudiant, Université Nazi BONI, Laboratoire d'étude et de recherche sur la fertilité du sol, Burkina Faso 01 BP 1091 Bobo-Dioulasso 01 Burkina Faso. Tel: +226-70050886. Auteur correspondant : E-mail: arnozo2000@gmail.com

Juliane Dao : PhD, Allemande, Universität Kassel, Organic Plant Production and Agroecosystems Research in the Tropics and Subtropics (OPATS) Steinstrasse 19, D-37213 Witzenhausen Germany, E-mail: juliane.dao@giz.de

Jean Pascal Désiré Lompo : PhD, Maître assistant, Burkinabè, Université de Dédougou (UDDG), Burkina Faso BP 176 Dédougou, E-mail : lompodesire@yahoo.fr

Delwendé Innocent Kiba : Doctorat Unique, Maître de recherches, Burkinabè, Département Gestion des Ressources Naturelles et Systèmes de Production, Centre National de la Recherche Scientifique et Technologique, Institut de l'Environnement et de Recherches Agricoles (INERA), Burkina Faso BP 476 Ouagadougou 01, E-mail : innokiba@yahoo.fr

Zacharia Gnankambary : PhD, Maître de recherches, Burkinabè, Département Gestion des Ressources Naturelles et Systèmes de Production, Centre National de la Recherche Scientifique et Technologique, Institut de l'Environnement et de Recherches Agricoles (INERA), Burkina Faso BP 476 Ouagadougou 01, E-mail : gnank_zach@hotmail.com

Papaoba Michel Sedogo : Doctorat d'Etat, Directeur de recherches, Burkinabè, Département Gestion des Ressources Naturelles et Systèmes de Production, Centre National de la Recherche Scientifique et Technologique, Institut de l'Environnement et de Recherches Agricoles (INERA), Burkina Faso BP 476 Ouagadougou 01,E-mail : michel_sedogo@yahoo.fr

Hassan Bismarck Nacro : Doctorat Unique, Professeur titulaire, Burkinabè, Université Nazi BONI, Laboratoire d'étude et de recherche sur la fertilité du sol, Burkina Faso 01 BP 1091 BoboDioulasso 01, E-mail : nacrohb@yahoo.fr

DOI: $10.25518 / 2295-8010.1935$

\section{Résumé :}

L'irrigation reste la principale source d'alcalinisation des sols. L'amendement gypse constitue une solution à ce processus de dégradation et d'amélioration de la productivité des cultures. 
Effets résiduel et d'apport continu de gypse sur les propriétés chimiques d’u...

Pour prévenir l'utilisation abusive du gypse en culture maraîchère, l'impact des modes d'apport du gypse a été étudié comparativement à la pratique paysanne (PP) sur un Lixisol dans un dispositif expérimental en split plot. Les traitements ont consisté à (i) modes d'apport du gypse avec deux niveaux : effet résiduel ( $\mathrm{GrA}$ ) et apport continu $(\mathrm{GrC})$ et à (ii) niveau de dégradation du sol avec deux niveaux : moyennement dégradé et fortement dégradé. Les résultats montrent que les modes d'apport ont abaissé le pH de 1,36 unité et 1,51 unité respectivement dans les sols moyennement dégradés et fortement dégradés. La plus forte baisse étant observée avec GrC. Pour la concentration en sodium échangeable, seul $\mathrm{GrC}$ a induit une baisse de 54,8 \% dans les sols moyennement dégradés. Dans les sols fortement dégradés, il a été abaissé de $87 \%$ et de $76 \%$ respectivement avec GrA et GrC comparativement à PP. Dans les sols fortement dégradés, les rendements obtenus avec GrA et $\mathrm{GrC}$ sont de 4 à 3,6 fois ceux de la PP respectivement.

Mots-clés : rendements, modes d’apport de Gypse, Lixisol

\section{Abstract : \\ Residual and repeated gypsum application effects on alkalinized soil chemical properties and maize (Zea mays L.) yields.}

Irrigation remains the main source of soil alkalization. Gypsum amendment is a solution to this process of degradation and improvement of crop productivity. To prevent the misuse of gypsum in urban agriculture, the impact of gypsum application modes was studied compared to farmer practice (PP) on Lixisol in a split plot design. The treatments consisted of (i) modes of applications of gypsum with two levels: residual effect (GrA); and continuous application ( $\mathrm{GrC})$ and (ii) level of soil degradation with two levels: moderately degraded and highly degraded. The results show that the modes of application decreased the $\mathrm{pH}$ by 1.36 units and 1.51 units respectively in moderately degraded and highly degraded soils. The highest decrease was observed with GrC. For soil exchangeable sodium concentration, only $\mathrm{GrC}$ induced a decrease of $54.8 \%$ in moderately degraded soils. In highly degraded soils, it was reduced by $87 \%$ and $76 \%$ respectively with GrA and GrC compared to PP. In highly degraded soils, the yields obtained with GrA and GrC are 4 to 3.6 times those of PP respectively.

Keywords : yields, modes of Gypsum application, Lixisol

\section{Introduction}

En Afrique subsaharienne, le recours à l'irrigation pour satisfaire la demande alimentaire et assurer les revenus nécessaires à la subsistance des populations en pleine croissance entraîne parfois des répercussions néfastes sur la durabilité des agrosystèmes. En effet, dans cette zone, la mise en œuvre de projets de plaines irriguées vise le plus souvent à résoudre le problème de manque d'eau (pénurie d'eau) que de sa qualité (Qadir, 2016). Aussi, comme les stations d'épuration n'existent pas ou sont inadéquates ou peu fonctionnelles en raison des coûts plus ou moins élevés pour leur construction et leur entretien (Koné, 2010), les eaux usées non traitées ou sommairement traitées des villes d'Afrique subsaharienne caractérisées par de fortes teneurs en sels solubles (Dao, 2017; Sou, 2009) sont déversées dans les cours d'eaux ou dans des réservoirs ou encore utilisées pour produire des cultures fraîches (Drechsel et al., 2006).

Dans ce dernier cas, ces pratiques modifient la qualité des sols. Elles conduisent parfois à la réduction 
de leur fertilité notamment par les processus d'alcalinisation résultant de l'accumulation des sels hydrosolubles $\left(\mathrm{K}^{+}, \mathrm{Mg}^{2+}, \mathrm{Ca}^{2+}, \mathrm{Cl}^{-}, \mathrm{SO}_{4}{ }^{--}, \mathrm{CO}_{3}{ }^{--}, \mathrm{HCO}_{3}{ }^{-}\right.$et $\left.\mathrm{Na}^{+}\right)$provenant principalement de l'eau d'irrigation. Ces processus de dégradation du sol largement répertoriés dans les zones arides et semi-arides affectent plus de $20 \%$ des terres irriguées dans le monde (Mahanta et al., 2015; Qadir, 2016). En conséquence, la productivité des terres baisse, entraînant leur abandon par les agriculteurs. Cela nécessite alors de trouver des solutions pour améliorer la gestion des systèmes irrigués, notamment par la restauration des sols alcalins-sodiques affectés.

L'efficacité des amendements directs du gypse dans l'amélioration des propriétés physico-chimiques (Armstrong \& Tanton, 1992; Chun et al., 2001; Kitila et al., 2020) et microbiologiques (Carter, 1986; Schultz et al., 2017; Wong et al., 2008) des sols salins-sodiques affectés ainsi que sur la croissance et les rendements des cultures (Abrol et al., 1988; Dao, 2017) a été prouvée par de nombreuses études. En effet, l'application du gypse $\left(\mathrm{CaSO}_{4}, 2 \mathrm{H}_{2} \mathrm{O}\right)$ aux sols salins-sodiques libère les ions $\mathrm{Ca}^{2+}$ dans la solution du sol qui remplacent les ions $\mathrm{Na}^{+}$des colloïdes du sol. Ces ions $\mathrm{Na}^{+}$sont précipités sous formes de sulfate de sodium $\left(\mathrm{Na}_{2} \mathrm{SO}_{4}\right)$ et de carbonates et bicarbonates de sodium $\left(\mathrm{NaHCO}_{3}\right.$ et $\left.\mathrm{Na}_{2} \mathrm{CO}_{3}\right)$ qui seront lessivés en présence d'eau. Le lessivage des ions $\mathrm{Na}^{+}$diminue les risques d'alcalinisation ainsi que le $\mathrm{pH}$ dont la réduction est encore induite par la présence des ions sulfates (Mao et al., 2016; Zhao et al., 2018).

Les ions $\mathrm{Ca}^{2+}$ améliorent la structure du sol grâce aux liaisons cationiques avec les particules d'argiles et la matière organique du sol. Cela augmente l'aération et la rétention d'eau du sol qui sont des facteurs stimulants de la vie et de l'activité microbienne (Chaganti \& Crohn, 2015; Gharaibeh et al., 2009; Schultz et al., 2017). Suivant la FAO (Ayers \& Westcot, 1976) et Rasouli et al. (2013), les doses optimales du gypse sont raisonnées en fonction de la concentration du sol en sodium échangeable. L'utilisation du gypse pour la remédiation des sols alcalins-sodiques affectés par l'irrigation des eaux usées en agriculture urbaine et périurbaine est très récente ; et il existe peu d'étude sur l'impact des arrières effets et de suppléments apports en doses recommandées de gypse sur les propriétés des sols et sur la productivité des cultures. Selon Elrashidi et al. (2010), les amendements supplémentaires de gypse réduisent la fertilité des sols et leur productivité même en présence de fertilisants organiques et minéraux en raison de la réduction de la solubilité des nutriments essentiels. À l'opposé, Clark et al. (2001) et Zhao et al. (2018) trouvent qu'en plus du rôle amendant aux sols agricoles, le gypse jouerait aussi un rôle fertilisant pour les cultures par la libération du calcium, du soufre et du magnésium qu'il contient.

Appréhender l'impact des modes d'apport des doses requises de gypse est primordial pour la gestion durable de la fertilité des terres irriguées. Dans cette étude, nous avons investigué en milieu paysan; sur les rôles de deux modes d'apport des doses requises de gypse (effet résiduel et apport continu effectué durant deux ans), sur (i) le maintien de la fertilité chimique des sols alcalins-sodiques et sur (ii) les rendements et (iii) les exportations de nutriments de la biomasse et des grains du maïs. L'objectif est de contribuer à une meilleure gestion des amendements des doses de gypse utilisées dans la remédiation des sols alcalinisés-sodiques affectés par l'irrigation des jardins maraichers urbains et périurbains avec les eaux usées prétraitées par lagunage. Nous partons des hypothèses que l'effet résiduel de la dose requise de gypse et l'application continue pendant deux ans de cette dose requise de gypse améliorent les propriétés chimiques du sol et les performances des cultures. 


\section{Matériel et méthodes}

\section{Zone d'étude et site d'expérimentation}

Létude a été conduite à Ouagadougou au Burkina Faso, sur le site maraicher urbain de Kossodo $\left(12^{\circ} 2^{\prime} 44,277^{\prime \prime} \mathrm{N}\right.$ et $1^{\circ} 28^{\prime} 22,394^{\prime \prime} \mathrm{O}, 300 \mathrm{~m}$ altitude). Ce site maraîcher dispose d'un aménagement hydroagricole à l'aval d'une station de traitement des eaux usées industrielles et urbaines (STEP). La STEP est conçue pour traiter $66 \%$ en volume et $72 \%$ en charge de polluants des eaux usées d'origine industrielles (brasserie, abattoir et cimenterie, huilerie, fer, acier, usines de tôles, hôtelleries). Elle est un lagunage à microphyte constitué de trois bassins anaérobies reliés de manière parallèle à deux bassins facultatifs. Ces bassins facultatifs sont reliés à trois bassins de maturation qui fonctionnent en parallèle.

L'aménagement couvre 35 ha de superficie irriguée avec les eaux usées prétraitées par le système de lagunage de la STEP. Cependant, le sol classé de Lixisol (WRB, 2015) et caractérisé par des teneurs faibles en argiles (29 \% sur l'horizon 0-50 cm) de type kaolinite (Dao, 2017; Sou, 2009) s'est rapidement déstructuré en l'espace de cinq années de production à cause des dépôts de sels de sodium contenus dans les eaux usées prétraitées entrainant l'abandon des champs par les producteurs. Le profil pédologique du sol du site indique trois horizons dont deux $(0 \mathrm{~cm} \leq \mathrm{H} 1<25$ $\mathrm{cm}$ et $45 \mathrm{~cm}<\mathrm{H} 3<120 \mathrm{~cm}$ ) sont caractérisés par une texture argilo limoneuse et un pH basique tandis que l'horizon H2 (25 à $45 \mathrm{~cm}$ ) présente une texture sableuse, un pH acide et une CEC faible (Sou, 2009).

\section{Dispositif expérimental}

Létude a été conduite sur un dispositif expérimental implanté depuis Mai 2015 et étant, au départ, un split-split plot avec quatre répétitions. Les parcelles ont subi le même historique cultural depuis 2015. Ensuite, ce dispositif a subi une modification en split plot au cours de la présente étude. Le premier facteur est le niveau de dégradation du sol (SDL) classé en fonction du pH du sol (Dao, 2017). Il comporte deux niveaux : Moyennement dégradé $(7<\mathrm{pH} \leq 8)$ et Fortement dégradé $(\mathrm{pH}>$ 8). Le deuxième facteur est le mode d'apport des doses requises de gypse avec trois niveaux : sans apport de gypse (pratique paysanne), effet résiduel ou apport de la dose requise de gypse à l'année n-1 en 2015 (GrA) et apport continu pendant deux ans (année n-1 et année n) en 2015 et en 2016 de la même dose requise de gypse (GrC).

La dose requise de gypse a été déterminée suivant la formule de la FAO (Ayers \& Westcot, 1976) et reprise par Rasouli et al. (2013). Elle équivaut à $12 \mathrm{t} \mathrm{ha}^{-1}$ de gypse pour les sols fortement dégradés et $8 \mathrm{t} \mathrm{ha}^{-1}$ de gypse pour les sols moyennement dégradés. Le gypse utilisé est en granules de diamètre inférieur à $1 \mathrm{~mm}$ avec une pureté de $85 \%$. C'est du plâtre principalement destiné à la construction de bâtiments et donc disponible dans le commerce et facilement accessible aux producteurs. Il est épandu et incorporé dans la profondeur de 0-20 cm du sol. Les parcelles d'application du gypse concernées dans cette étude ont été irriguées pendant la saison agricole sèche de 2015-2016 (octobre 2015 à avril 2016) avec de l'eau propre provenant d'une borne fontaine de la desserte domestique du système de distribution de l'Office National de l'Eau et de l'Assainissement (ONEA) du Burkina Faso. 


\section{Pratiques culturales}

L'expérimentation est conduite en 2016 au cours de la saison pluvieuse (Juin à Octobre) avec la culture de maïs (Zea mays L,), connue pour sa sensibilité au sodium échangeable du sol (Abrol et al., 1988). C'est la variété composite K.E.J. (Kamboinsé Extra Précoce Jaune) qui a été utilisée pour son adaptation à des pluviosités moyennes annuelles de 500 à $900 \mathrm{~mm}$ de pluie (CEDEAO et al., 2016). Sur toutes les parcelles, un labour d'environ $15 \mathrm{~cm}$ de profondeur suivi de l'application de fumier de bovins $\left(10,24 \mathrm{t} \mathrm{ha}^{-1}\right)$ a été réalisé. Les engrais NPK-SB (14-23-14-6-1) à raison de 150 $\mathrm{kg} \mathrm{ha}^{-1}$ et l'urée $(46 \% \mathrm{~N})$ à la dose de $100 \mathrm{~kg} \mathrm{ha}^{-1}$ ont été appliqués sur toutes les parcelles. Le NPK-SB a été appliqué au $16^{\text {èm }}$ jour après le semis. L'urée a été appliquée en deux tranches égales au $23^{\text {èm }}$ et au $40^{\text {èm }}$ jour après le semis. Le traitement des plants au $44^{\text {èm }}$ jour après le semis avec l'insecticide D-Cis a permis de maîtriser l'attaque des chenilles défolliatrices et foreuses des tiges de maïs.

\section{Échantillonnage du fumier, des sols et du matériel végétal}

Les échantillons du fumier ont été prélevés au moment de l'application en cinq points différents à l'intérieur du tas homogénéisé. Un échantillon composite de sol formé de cinq points de prélèvement à la profondeur de $0-10 \mathrm{~cm}$ a été réalisé à la récolte pour chaque traitement. Les échantillons de la paille et des grains du maïs ont été collectés à la récolte. Ils proviennent des plantes issues des quatre poquets de semis centraux du carré de rendement de la parcelle. Ces échantillons ont été d'abord séchés à l'ombre puis à l'étuve à $60^{\circ} \mathrm{C}$ jusqu'à poids constant.

\section{Performances du maïs}

Les rendements paille et grains du maïs ont été déterminés. Les exportations des nutriments azote $(\mathrm{N})$, phosphore $(\mathrm{P})$, potassium $(\mathrm{K})$, calcium $(\mathrm{Ca})$ et sodium $(\mathrm{Na})$ par la paille et les grains ont été calculées en multipliant leurs teneurs par les rendements.

\section{Analyses chimiques}

Les teneurs en carbone total (C-total) et azote total (N-total) des échantillons de fumier, de la paille et des grains du maïs et des sols ont été déterminées par voie de combustion sèche selon la méthode DUMAS à l'aide de l'auto-analyseur C/N Vario MAX (Elementar Analysensysteme GmbH, Langenselbold, Allemagne). Pour la détermination des teneurs en phosphore (P), en potassium (K), en calcium $(\mathrm{Ca})$ et en sodium $(\mathrm{Na})$, les échantillons ont été d'abord calcinés à $550^{\circ} \mathrm{C}$ pendant 2 heures, puis des dosages ont été faits à partir des filtrats de cendres minéralisés à l'acide chlorhydrique ( $\mathrm{HCl}$ ) suivant les normes du VDLUFA (Naumann \& Bassler, 1976). Le phosphore total (P-total) a été dosé par spectrophotométrie (Hitachi U-2000, Hitachi Ltd, Tokyo, Japon) à 460 nm. Le potassium total (K-total), le Na et le Ca ont été dosés par photométrie de flamme (BWB-XP Technologies Ltd, BWB Technology UK Ltd, Halstead, Essex, Royaume-Uni).

Le phosphore disponible du sol, a été déterminé suivant la méthode Olsen (Olsen et al., 1954).

Le pH eau des sols a été mesuré en utilisant un pH-mètre électrométrique dans une suspension de sol eau au rapport $1: 2,5$ (Pansu \& Gautheyrou, 2006). La CEC effective et les bases échangeables des sols à savoir le sodium échangeable $\left(\mathrm{Na}^{+}\right.$échangeable), le calcium échangeable $\left(\mathrm{Ca}^{2+}\right.$ 
Effets résiduel et d’apport continu de gypse sur les propriétés chimiques d'u...

échangeable), le potassium échangeable $\left(\mathrm{K}^{+}\right.$échangeable) et le magnesium échangeable $\left(\mathrm{Mg}^{2+}\right.$ échangeable) ont été déterminées par la méthode du chlorure de cobalt hexamine (Ciesielski \& Sterckeman, 1997).

\section{Analyses statistiques}

Le modèle linéaire à effets mixtes estimé par la méthode du maximum de vraisemblance restreinte a été utilisé pour déterminer l'influence du niveau de dégradation du sol, des modes d'apport des doses requises de gypse ainsi que leurs combinaisons sur les paramètres évalués.

Le logiciel d'analyse statistique R version 4.0.2 (R Core Team, 2018) et le package lme4 (Bates et al., 2015) de la fonction glmer ont été utilisés à cet effet. Les champs des producteurs ont été considérés comme les effets aléatoires du model linéaire mixte. Les valeurs de $\mathrm{p}$ pour les $\mathrm{t}$ tests utilisés ont été obtenues par approximation de la méthode de Satterthwaite et de la fonction lmerModLmerTest (Kuznetsova et al., 2017). Sur le modèle ajusté, un test d'analyse des variances ANOVA à deux facteurs a été réalisé pour comparer l'effet de chaque facteur (niveau de dégradation $\mathrm{du}$ sol, modes d'apport des doses requises de gypse) et de leurs termes d'interactions. Les comparaisons par pair de ces moyennes ont été faites par la méthode de Tukey grâce au package multcompView. La séparation des moyennes statistiquement significatives a été faite au seuil de 5 \% de probabilité. Les analyses statistiques ont été effectuées avec le programme R ( 4 4.0.2.lnk) en utilisant l'interface RStudio version 1.3.1093 et les progiciels multicomp (Hothorn et al., 2008) et nlme (Pinheiro et al., 2018).

\section{Résultats}

\section{pH et concentration en sodium échangeable du sol}

Les modes d'apport des doses de gypse ont induit des baisses du $\mathrm{pH}$ et de la concentration en $\mathrm{Na}^{+}$échangeable du sol. Quelle que soit le niveau de dégradation du sol, le pH a baissé avec l'application du gypse par rapport à la pratique paysanne. L’apport du gypse a abaissé le pH de 1,36 unités et 1,51 unités respectivement dans les sols moyennement dégradés et fortement dégradés. La plus forte baisse du $\mathrm{pH}$ a été observée avec l'application continue des doses de gypse. Pour la concentration du sol en $\mathrm{Na}^{+}$échangeable, seul le mode $\mathrm{GrC}$ a induit une baisse de 54,8 \% sur les sols moyennement dégradés. Par contre sur les sols fortement dégradés, les traitements GrA et GrC ont induit des baisses de $87 \%$ et de $76 \%$ respectivement par rapport à la pratique paysanne.

\section{Teneurs en carbone total et en nutriments totaux du sol}

Les modes d'apport du gypse ont augmenté les teneurs en C-total, N-total et réduit celles du K-total par rapport à la pratique paysanne (Tableau 1 et Tableau 2). Dans les sols moyennement dégradés, le mode GrA présente la plus forte teneur en C-total $\left(16,2 \pm 3,4 \mathrm{~g} \mathrm{~kg}^{-1}\right.$ sol) et en N-total $(1575,9$ $\pm 375,1 \mathrm{mg} \mathrm{kg}^{-1} \mathrm{sol}$ ) par rapport à $\mathrm{PP}$ et à $\mathrm{GrC}$ qui présentent des valeurs similaires. Pour les sols fortement dégradés, les modes d'apport du gypse n’ont pas induit de différence significative sur les teneurs en C-total et $\mathrm{N}$-total du sol par rapport à la pratique paysanne. Les teneurs en P-total du sol de la PP sont similaires à celles de GrA et GrC quel que soit le niveau de dégradation du sol. Indépendamment du niveau de dégradation du sol, le K-total a subi des baisses de $45 \%$ avec le 
mode GrA et de $62 \%$ avec le mode GrC comparativement à la pratique paysanne (PP) d'une part et de $31 \%$ avec le mode $\mathrm{GrC}$ par rapport à l'effet résiduel des doses de gypse (GrA) d'autre part.

Tableau 1 : Valeurs $F$ et niveau de signification à partir de l'ANOVA à deux facteurs comparant les paramètres chimiques du sol

\begin{tabular}{|c|c|c|c|c|c|c|c|c|c|c|c|}
\hline $\begin{array}{l}\text { Source de } \\
\text { variation }\end{array}$ & $\mathrm{df}$ & $\mathrm{pH}$ & $\begin{array}{l}\text { C- } \\
\text { total }\end{array}$ & $\begin{array}{l}\mathrm{N}- \\
\text { total }\end{array}$ & $\begin{array}{l}\text { P- } \\
\text { total }\end{array}$ & K-total & $\begin{array}{l}\text { P- } \\
\text { Olsen }\end{array}$ & $\begin{array}{l}\mathrm{Na} \\
\text { échan. }\end{array}$ & $\begin{array}{l}\text { Ca } \\
\text { échan }\end{array}$ & $\begin{array}{l}\text { CEC } \\
\text { effective }\end{array}$ & SB \\
\hline NDS & 1 & $3,13^{\mathrm{ns}}$ & $\begin{array}{l}2,12 \\
\mathrm{~ns}\end{array}$ & $\begin{array}{l}1,57 \\
\mathrm{~ns}\end{array}$ & $\begin{array}{l}0,05 \\
\mathrm{~ns}\end{array}$ & $0,28^{\mathrm{ns}}$ & $5,36 *$ & $5,62^{\mathrm{ns}}$ & $5,42^{*}$ & $0,01^{\mathrm{ns}}$ & $5,48^{*}$ \\
\hline MoG & 2 & $268,32 * * *$ & $8,03^{*}$ & $7,33^{*}$ & $5,09 *$ & $82,28 * * *$ & $4,63 *$ & $33,66^{* * *}$ & $41,53 * * *$ & $4,43 *$ & $13,53 * *$ \\
\hline NDS x MoG & 2 & $3,73 *$ & $\begin{array}{l}3,89 \\
n s\end{array}$ & $\begin{array}{l}3,33 \\
n s\end{array}$ & $\begin{array}{l}0,03 \\
n s\end{array}$ & $0,20^{\mathrm{ns}}$ & $\begin{array}{l}0,65 \\
n s\end{array}$ & $2,54^{\mathrm{ns}}$ & $1,89^{\mathrm{ns}}$ & $0,31 \mathrm{~ns}$ & $2,54^{\mathrm{ns}}$ \\
\hline
\end{tabular}

NDS $=$ Niveau de dégradation $\mathrm{du}$ sol $; \mathrm{MoG}=$ modes d'apport du gypse $; \mathrm{ns}=$ non significatif $; *=\mathrm{P}<0,05 ;{ }^{* *}=\mathrm{P}<$ 0,$01 ; * * *=\mathrm{P}<0,001$

\section{Bases échangeables et P-Olsen du sol}

Les modes d'apport du gypse ont augmenté la concentration en $\mathrm{Ca}^{2+}$ échangeable du sol par rapport à la pratique paysanne (PP) (Tableau 1 et Tableau 2). Les concentrations du $\mathrm{Ca}^{2+}$ échangeable observées avec le mode $\mathrm{GrC}$ sont 1,67 et 2,2 fois celles de la pratique paysanne respectivement dans les sols moyennement dégradés et fortement dégradés. Les modes d'apport du gypse augmentent également la somme des bases échangeables du sol par rapport à la pratique paysanne (Tableau 1 et Tableau 2). Sur les sols fortement dégradés, la somme des bases échangeables du sol a augmenté de 36,24 $\pm 4,23 \%$ (PP) à 48,83 $\pm 4,01 \%$ (GrC). Quel que soit le niveau de dégradation du sol, les modes d'apport des doses de gypse n'améliorent pas la CEC effective.

Quant à la teneur en P-Olsen du sol, elle a augmenté de $40 \%$ avec l'application de GrC uniquement sur les sols fortement dégradés (Tableau 1 et Tableau 2). 
Effets résiduel et d’apport continu de gypse sur les propriétés chimiques d’u...

Tableau 2 : Valeurs moyennes et écart type (en parenthèses) des propriétés chimiques du sol à la profondeur 0-10 cm suivant les traitements appliqués dans les sols moyennement dégradés et dans les sols fortement dégradés.

\begin{tabular}{|c|c|c|c|c|c|c|c|c|c|}
\hline \multirow[b]{2}{*}{ Paramètres } & \multirow[b]{2}{*}{ Unité } & \multicolumn{4}{|c|}{ Sols moyennement dégradés } & \multicolumn{4}{|c|}{ Sols fortement dégradés } \\
\hline & & $\mathrm{PP}$ & GrA & $\mathrm{GrC}$ & $\begin{array}{l}\text { Valeur } \\
\text { de } p\end{array}$ & $\mathrm{PP}$ & GrA & $\mathrm{GrC}$ & $\begin{array}{l}\text { Valeur } \\
\text { de p }\end{array}$ \\
\hline $\mathrm{pH}$ & & $\begin{array}{l}9,10 \\
(0,40) \mathrm{a}\end{array}$ & $\begin{array}{l}7,74 \\
(0,32) \mathrm{b}\end{array}$ & $\begin{array}{l}7,42 \\
(0,24) \mathrm{c}\end{array}$ & $<0,001$ & $\begin{array}{l}9,17 \\
(0,47) \mathrm{a}\end{array}$ & $\begin{array}{l}8,01 \\
(0,09) \mathrm{b}\end{array}$ & $\begin{array}{l}7,66 \\
(0,12) \mathrm{c}\end{array}$ & 0 \\
\hline C-total & $\mathrm{g} \mathrm{kg}^{-1}$ & $\begin{array}{l}11,10 \\
(1,83) \mathrm{a}\end{array}$ & $\begin{array}{l}16,22 \\
(3,39) \mathrm{b}\end{array}$ & $\begin{array}{l}14,05 \\
(3,67) a\end{array}$ & 0,01 & $\begin{array}{l}9,85 \\
(2,23)\end{array}$ & $\begin{array}{l}12,85 \\
(2,20)\end{array}$ & $\begin{array}{l}13,14 \\
(3,12)\end{array}$ & 0,08 \\
\hline N-total & & $\begin{array}{l}1,11 \\
(0,13) a b\end{array}$ & $\begin{array}{l}1,58 \\
(0,37) \mathrm{b}\end{array}$ & $\begin{array}{l}1,36 \\
(0,39) \mathrm{a}\end{array}$ & 0,01 & $\begin{array}{l}0,99 \\
(0,25)\end{array}$ & $\begin{array}{l}1,25 \\
(0,20)\end{array}$ & $\begin{array}{l}1,27 \\
(0,27)\end{array}$ & 0,13 \\
\hline P-total & $\mathrm{mg} \mathrm{kg}^{-1}$ & $454(58)$ & $568(69)$ & $533(108)$ & 0,13 & $473(69)$ & $599(150)$ & $561(172)$ & 0,09 \\
\hline K-total & & $\begin{array}{l}377(125) \\
\mathrm{a}\end{array}$ & $\begin{array}{l}197(45) \\
b\end{array}$ & $142(53) \mathrm{c}$ & $<0,001$ & $\begin{array}{l}325(48) \\
\mathrm{a}\end{array}$ & $\begin{array}{l}191(24) \\
b\end{array}$ & $125(27) \mathrm{c}$ & $<0,001$ \\
\hline P-Olsen. & & $108(26)$ & $132(24)$ & $124(23)$ & 0,34 & $83(20)$ a & $\begin{array}{l}110(13) \\
\mathrm{ab}\end{array}$ & $\begin{array}{l}116(17) \\
b\end{array}$ & 0,02 \\
\hline Na échan. & $\begin{array}{l}\mathrm{Cmol}(+) \\
\mathrm{kg}^{-1}\end{array}$ & $\begin{array}{l}2,88 \\
(1,75) \mathrm{a}\end{array}$ & $\begin{array}{l}1,09 \\
(0,21) a b\end{array}$ & $\begin{array}{l}1,19 \\
(1,15) b\end{array}$ & 0,02 & $\begin{array}{l}2,03 \\
(0,86) \mathrm{a}\end{array}$ & $\begin{array}{l}0,27 \\
(0,20) \mathrm{b}\end{array}$ & $\begin{array}{l}0,48 \\
(0,13) b\end{array}$ & $<0,001$ \\
\hline Ca échan. & & $\begin{array}{l}15,99 \\
(3,62) a\end{array}$ & $\begin{array}{l}17,58 \\
(3,86) \mathrm{a}\end{array}$ & $\begin{array}{l}26,79 \\
(1,49) \mathrm{b}\end{array}$ & $<0,001$ & $\begin{array}{l}15,41 \\
(2,33) \mathrm{a}\end{array}$ & $\begin{array}{l}21,65 \\
(2,73) a\end{array}$ & $\begin{array}{l}34,07 \\
(7,40) \mathrm{b}\end{array}$ & $<0,001$ \\
\hline $\begin{array}{l}\text { CEC } \\
\text { effective }\end{array}$ & & $\begin{array}{l}83,3 \\
(20,2)\end{array}$ & $\begin{array}{l}82,9 \\
(17,3)\end{array}$ & $\begin{array}{l}95,5 \\
(11,2)\end{array}$ & 0,24 & $80,2(8,9)$ & $87,5(9,7)$ & $96(14,0)$ & 0,13 \\
\hline SB & $\%$ & $\begin{array}{l}36,40 \\
(4,65)\end{array}$ & $\begin{array}{l}35,53 \\
(5,53)\end{array}$ & $\begin{array}{l}40,36 \\
(3,81)\end{array}$ & 0,13 & $\begin{array}{l}36,24 \\
(4,23) \mathrm{a}\end{array}$ & $\begin{array}{l}38,70 \\
(2,10) \mathrm{a}\end{array}$ & $\begin{array}{l}48,83 \\
(4,01) \mathrm{b}\end{array}$ & $<0,001$ \\
\hline
\end{tabular}

$\mathrm{PP}=$ Pratique paysanne, $\mathrm{GrA}=$ effet résiduel de la dose requise de gypse $; \mathrm{GrC}=$ apport continuel de la dose requise de gypse.

Dans les sols moyennement dégradés ou dans les sols fortement dégradés, les traitements suivis de la même lettre sur la même ligne ne sont pas statistiquement différents au seuil de $5 \%$ de probabilité.

\section{Rendements paille et grains du maïs}

Les modes d'apport du gypse ont augmenté les rendements paille et grains du maïs par rapport à la pratique paysanne (Tableau 3 et Tableau 4). Sur les sols fortement dégradés, les rendements paille et grains observés avec les modes d'apport du gypse sont de 4 et 3,6 fois ceux observés avec 
la pratique paysanne. Par contre, sur les sols moyennement dégradés, l'apport du gypse n'améliore pas les rendements paille et grains par rapport à la pratique paysanne.

Tableau 3 : Valeurs $F$ et niveau de signification à partir de l'ANOVA à deux facteurs comparant les rendements et les exportations de nutriments par le maïs

\begin{tabular}{|c|c|c|c|c|c|c|c|c|c|c|c|c|c|}
\hline & & \multicolumn{2}{|c|}{ Rendements } & \multicolumn{10}{|c|}{ Exportations de nutriments } \\
\hline & & & & $\mathrm{N}$ & & $\mathrm{P}$ & & K & & $\mathrm{Ca}$ & & $\mathrm{Na}$ & \\
\hline $\begin{array}{l}\text { Source } \\
\text { de } \\
\text { variation }\end{array}$ & $\mathrm{df}$ & Paille & Grains & Paille & Grains & Paille & Grains & Paille & Grains & Paille & Grains & Paille & Grains \\
\hline NDS & 1 & $0,11^{\mathrm{ns}}$ & $0,05^{\mathrm{ns}}$ & $\begin{array}{l}0,75 \\
\text { ns }\end{array}$ & $0,17^{\mathrm{ns}}$ & $0,67^{\mathrm{ns}}$ & $0,07^{\mathrm{ns}}$ & $1,43^{\mathrm{ns}}$ & $0,16^{\mathrm{ns}}$ & $2,39 * * *$ & $\begin{array}{l}0,22 \\
\text { ns }\end{array}$ & $\begin{array}{l}2,06 \\
\text { ns }\end{array}$ & $\begin{array}{l}0,02 \\
\text { ns }\end{array}$ \\
\hline MoG & 2 & $\begin{array}{l}19,29 \\
* * *\end{array}$ & $17,83^{* * *}$ & $4,16^{*}$ & $12,44^{* * *}$ & $11,44^{* * *}$ & $17,99 * * *$ & $11,94^{* * *}$ & $12,37 * * *$ & $0,85^{* *}$ & $\begin{array}{l}0,11 \\
\text { ns }\end{array}$ & $6,31 * *$ & $\begin{array}{l}0,19 \\
\text { ns }\end{array}$ \\
\hline $\begin{array}{l}\text { NDS x } \\
\text { MoG }\end{array}$ & 2 & $6,11^{*}$ & $5,38^{*}$ & $4,00 *$ & $3,73 *$ & $2,48^{\mathrm{ns}}$ & $5,36 *$ & $1,50^{\mathrm{ns}}$ & $4,21 *$ & $0,30^{\mathrm{ns}}$ & $\begin{array}{l}0,03 \\
\text { ns }\end{array}$ & $\begin{array}{l}1,29 \\
\text { ns }\end{array}$ & $\begin{array}{l}1,54 \\
\mathrm{~ns}\end{array}$ \\
\hline
\end{tabular}

NDS $=$ Niveau de dégradation du sol $;$ MoG $=$ modes d'apport du gypse $;$ ns $=$ non significatif $; *=$ $\mathrm{p}<0,05 ;{ }^{* *}=\mathrm{p}<0,01 ; * * *=\mathrm{p}<0,001$

\section{Exportations de nutriments par le maïs}

Les modes d'apport du gypse ont augmenté les exportations en éléments majeurs par la paille et par les grains du maïs (Tableau 3 et Tableau 4). 
Effets résiduel et d’apport continu de gypse sur les propriétés chimiques d’u...

Tableau 4 : Valeurs moyennes et écart type (en parenthèses) des rendements en paille et grains et des exportations de nutriments par le maïs suivant les traitements appliqués dans les sols moyennement dégradés et dans les sols fortement dégradés.

\begin{tabular}{|c|c|c|c|c|c|c|c|c|c|}
\hline \multirow[b]{2}{*}{ Paramètres } & & \multicolumn{4}{|c|}{ Sols moyennement dégradés } & \multicolumn{4}{|c|}{ Sols fortement dégradés } \\
\hline & & $\mathrm{PP}$ & GrA & $\mathrm{GrC}$ & $\begin{array}{l}\text { Valeur } \\
\text { de } \mathrm{p}\end{array}$ & PP & GrA & $\mathrm{GrC}$ & $\begin{array}{l}\text { Valeur } \\
\text { de } \mathrm{p}\end{array}$ \\
\hline \multirow[t]{2}{*}{ Rendements } & Paille & $\begin{array}{l}2,92 \\
(0,78)\end{array}$ & $\begin{array}{l}3,67 \\
(1,95)\end{array}$ & $\begin{array}{l}3,62 \\
(0,94)\end{array}$ & 0,4 & $\begin{array}{l}1,02 \\
(1,02) \mathrm{a}\end{array}$ & $\begin{array}{l}4,02 \\
(1,25) \mathrm{b}\end{array}$ & $\begin{array}{l}4,30 \\
(0,61) \mathrm{b}\end{array}$ & 0 \\
\hline & Grains & $\begin{array}{l}1,37 \\
(0,39)\end{array}$ & $\begin{array}{l}1,99 \\
(1,23)\end{array}$ & $\begin{array}{l}1,71 \\
(0,68)\end{array}$ & 0,28 & $\begin{array}{l}0,61 \\
(0,23) a\end{array}$ & $\begin{array}{l}2,05 \\
(0,58) \mathrm{b}\end{array}$ & $\begin{array}{l}2,19 \\
(0,28) \mathrm{b}\end{array}$ & $\begin{array}{l}< \\
0,001\end{array}$ \\
\hline \multirow[t]{5}{*}{$\begin{array}{l}\text { Exportations par la } \\
\text { paille }\left(\mathrm{kg} \mathrm{ha}^{-1}\right)\end{array}$} & $\mathrm{N}$ & $\begin{array}{l}41,60 \\
(11,34)\end{array}$ & $\begin{array}{l}42,66 \\
(19,04)\end{array}$ & $\begin{array}{l}38,23 \\
(13,74)\end{array}$ & 0,77 & $\begin{array}{l}16,51 \\
(17,97) \mathrm{a}\end{array}$ & $\begin{array}{l}47,14 \\
(19,04) \mathrm{b}\end{array}$ & $\begin{array}{l}36,16 \\
(6,43) \mathrm{ab}\end{array}$ & 0,05 \\
\hline & $\mathrm{P}$ & $\begin{array}{l}2,99 \\
(1,57)\end{array}$ & $\begin{array}{l}5,11 \\
(3,53)\end{array}$ & $\begin{array}{l}3,83 \\
(0,79)\end{array}$ & 0,33 & $\begin{array}{l}0,97 \\
(1,01) \mathrm{a}\end{array}$ & $\begin{array}{l}4,88 \\
(2,21) \mathrm{b}\end{array}$ & $\begin{array}{l}4,10 \\
(0,71) \mathrm{b}\end{array}$ & 0,01 \\
\hline & $\mathrm{K}$ & $\begin{array}{l}142,66 \\
(15,69)\end{array}$ & $\begin{array}{l}175,56 \\
(47,83)\end{array}$ & $\begin{array}{l}174,54 \\
(53,47)\end{array}$ & 0,22 & $\begin{array}{l}146,23 \\
(23,73) a\end{array}$ & $\begin{array}{l}216,19 \\
(8,87) \mathrm{b}\end{array}$ & $\begin{array}{l}190,14 \\
(35,47) \mathrm{ab}\end{array}$ & 0 \\
\hline & $\mathrm{Ca}$ & $\begin{array}{l}56,62 \\
(13,56) \mathrm{b}\end{array}$ & $\begin{array}{l}37,57 \\
(4,36) \mathrm{a}\end{array}$ & $\begin{array}{l}39,92 \\
(6,46) \mathrm{a}\end{array}$ & 0,05 & $\begin{array}{l}68,45 \\
(15,23)\end{array}$ & $\begin{array}{l}76,74 \\
(50,32)\end{array}$ & $\begin{array}{l}49,07 \\
(4,66)\end{array}$ & 0,35 \\
\hline & $\mathrm{Na}$ & $\begin{array}{l}18,70 \\
(7,55) \mathrm{b}\end{array}$ & $\begin{array}{l}3,50 \\
(2,21) \mathrm{a}\end{array}$ & $\begin{array}{l}3,44 \\
(3,15) \mathrm{a}\end{array}$ & 0 & $\begin{array}{l}11,72 \\
(16,39)\end{array}$ & $\begin{array}{l}3,42 \\
(3,73)\end{array}$ & $\begin{array}{l}2,23 \\
(0,89)\end{array}$ & 0,52 \\
\hline \multirow[t]{5}{*}{$\begin{array}{l}\text { Exportations par les } \\
\text { grains }\left(\mathrm{kg} \mathrm{ha}^{-1}\right)\end{array}$} & $\mathrm{N}$ & $\begin{array}{l}27,38 \\
(7,81)\end{array}$ & $\begin{array}{l}38,53 \\
(24,26)\end{array}$ & $\begin{array}{l}33,18 \\
(13,69)\end{array}$ & 0,56 & $\begin{array}{l}12,57 \\
(4,75) \mathrm{a}\end{array}$ & $\begin{array}{l}37,98 \\
(12,63) \mathrm{b}\end{array}$ & $\begin{array}{l}40,22 \\
(6,76) \mathrm{b}\end{array}$ & $\begin{array}{l}< \\
0,001\end{array}$ \\
\hline & $\mathrm{P}$ & $\begin{array}{l}2,83 \\
(0,83)\end{array}$ & $\begin{array}{l}3,94 \\
(2,44)\end{array}$ & $\begin{array}{l}3,58 \\
(1,42)\end{array}$ & 0,31 & $\begin{array}{l}1,23 \\
(0,47) \mathrm{a}\end{array}$ & $\begin{array}{l}4,20 \\
(1,27) \mathrm{b}\end{array}$ & $\begin{array}{l}4,38 \\
(0,59) \mathrm{b}\end{array}$ & $\begin{array}{l}< \\
0,001\end{array}$ \\
\hline & $\mathrm{K}$ & $\begin{array}{l}12,87 \\
(3,48)\end{array}$ & $\begin{array}{l}14,98 \\
(7,95)\end{array}$ & $\begin{array}{l}15,80 \\
(3,53)\end{array}$ & 0,45 & $\begin{array}{l}4,46 \\
(4,46) \mathrm{a}\end{array}$ & $\begin{array}{l}17,19 \\
(5,95) \mathrm{b}\end{array}$ & $\begin{array}{l}17,08 \\
(2,37) \mathrm{b}\end{array}$ & 0 \\
\hline & $\mathrm{Ca}$ & $\begin{array}{l}1,79 \\
(0,47) \mathrm{a}\end{array}$ & $\begin{array}{l}2,35 \\
(0,07) \mathrm{b}\end{array}$ & $\begin{array}{l}2,33 \\
(0,06) \\
a b\end{array}$ & 0,01 & $\begin{array}{l}2,44 \\
(0,06) \mathrm{a}\end{array}$ & $\begin{array}{l}3,33 \\
(0,55) \mathrm{b}\end{array}$ & $\begin{array}{l}2,50 \\
(0,05) \mathrm{a}\end{array}$ & 0 \\
\hline & $\mathrm{Na}$ & $\begin{array}{l}0,16 \\
(0,05)\end{array}$ & $\begin{array}{l}0,28 \\
(0,33)\end{array}$ & $\begin{array}{l}0,10 \\
(0,05)\end{array}$ & 0,43 & $\begin{array}{l}0,13 \\
(0,09)\end{array}$ & $\begin{array}{l}0,13 \\
(0,05)\end{array}$ & $\begin{array}{l}0,15 \\
(0,03)\end{array}$ & 0,45 \\
\hline
\end{tabular}

$\mathrm{PP}=$ Pratique paysanne, $\mathrm{GrA}=$ effet résiduel de la dose requise de gypse $; \mathrm{GrC}=$ apport continu de dose requise de gypse. 
la même ligne ne sont pas statistiquement différents au seuil de $5 \%$ de probabilité.

Sur les sols fortement dégradés, les modes d'apport du gypse ont induit une augmentation des exportations en N, $\mathrm{P}$ et $\mathrm{K}$ par la paille et par les grains comparativement à la pratique paysanne. L'augmentation de ces exportations avec les modes d'apport du gypse par rapport à la pratique paysanne sur les sols fortement dégradés correspond à $25,1 \mathrm{~kg} \mathrm{~N} \mathrm{ha}^{-1}$ pour la paille et à 26,5 $\mathrm{kg} \mathrm{N}$ ha ${ }^{-1}$ pour les grains du maïs. Pour celle du P, elle est 3,6 et 2,5 fois les exportations du $\mathrm{P}$ enregistrées par la pratique paysanne respectivement dans la paille et dans les grains. Quant à l'augmentation des exportations en $\mathrm{K}$ par les modes d'apport du gypse comparativement à la pratique paysanne sur les sols fortement dégradés, elle est de $57 \mathrm{~kg} \mathrm{ha}^{-1}$ pour la paille et de 2,84 fois plus importante dans les grains. Cependant, sur les sols moyennement dégradés, l'apport du gypse n'a pas d'effet.

Pour les exportations de $\mathrm{Ca}$ et de $\mathrm{Na}$ par la paille du maïs, les modes d'apport du gypse comparativement à la pratique paysanne (PP) ont induit des baisses de $18 \mathrm{~kg} \mathrm{Ca} \mathrm{ha}^{-1}$ et de 15,23 kg $\mathrm{Na} \mathrm{ha}^{-1}$ sur les sols moyennement dégradés. Par contre dans les grains récoltés, les exportations de Ca ont augmenté de $24 \%$ et de 36,5\% avec le mode GrA par rapport à la pratique paysanne (PP) respectivement sur les sols moyennement dégradés et sur les sols fortement dégradés.

\section{Discussions}

\section{Effets des modes d'apport du gypse sur les propriétés chimiques du sol.}

Les baisses du $\mathrm{pH}$ et de la concentration en $\mathrm{Na}^{+}$échangeable du sol avec l'application des modes d'apport du gypse par rapport à la pratique paysanne résultent des ions $\mathrm{Ca}^{2+}$ libérés par la dissolution du gypse (Gharaibeh et al., 2014; Qadir et al., 2001; Rengasamy, 1987). En effet, la hausse de la concentration du sol en $\mathrm{Ca}^{2+}$ échangeable résultant des modes d'apport du gypse par rapport à la pratique paysanne (Tableau 1 et Tableau 2) peut être attribuée à l'augmentation des ions $\mathrm{Ca}^{2+}$ dans la solution du sol ; ce qui induit le déplacement et le remplacement des ions $\mathrm{Na}^{+}$des colloïdes du sol vers la solution du sol facilitant ainsi leur lessivage (Mahmoodabadi et al., 2013).

Les ions $\mathrm{Na}^{+}$dans la solution du sol sont précipités sous forme de sulfate de sodium $\left(\mathrm{Na}_{2} \mathrm{SO}_{4}\right)$ (Zhao et al., 2018), entrainant alors la réduction de l'alcalinité du sol (Gharaibeh et al., 2014). Kim et al. (2017) sur les sols limoneux des côtes Sud-Coréennes, Clark et al. (2007) sur les Sodosols d'Australie et Makoi \& Ndakidemi (2007) dans les plaines alluvionnaires de la Tanzanie ont trouvé des résultats similaires par l'application du gypse. En effet, Kim et al. (2017) ont obtenu des baisses de la concentration du sol en $\mathrm{Na}^{+}$échangeable équivalentes à $78,6 \%$ et à $71,4 \%$ respectivement avec l'application du gypse seul et l'application du gypse + compost de paille de riz après une année de culture. Clark et al. (2007) ont enregistré une baisse du pourcentage en $\mathrm{Na}^{+}$échangeable du sol de 51 \% par l'amendement de gypse après une année de culture. Dans les plaines sucrières de la Tanzanie, Makoi \& Ndakidemi (2007) après deux années d'apport du gypse ont relevé une baisse de $86,6 \%$ pour le pourcentage en $\mathrm{Na}^{+}$échangeable du sol.

Par contre, nos résultats sont 3 à 4 fois et de 2,6 à 9,6 fois supérieurs à ceux rapportés par Zhao et al. (2018) et par Mao et al. (2016) qui ont travaillé avec du gypse issu de la désulfurisation 
Effets résiduel et d'apport continu de gypse sur les propriétés chimiques d'u...

des fumées de gaz. Zhao et al. (2018) ont relevé une baisse de $18,7 \%$ du pourcentage en $\mathrm{Na}^{+}$ échangeable du sol une année après amendement du gypse aux sols Solonetz de la partie SudOuest de la plaine Songnen en Chine. Mao et al. (2016) en appliquant $15 \mathrm{t} \mathrm{ha}^{-1}$ de ce gypse aux sols marins-côtiers de la partie Sud-est de la Chine ont abaissé le pH du sol de l'horizon 0-10 cm de $9,3 \%$ et de $21 \%$ après respectivement 6 mois et 18 mois de mise en culture. Quant à la réduction du pH des sols alcalins-sodiques, Gharaibeh et al. (2014) souligne qu'elle est généralement liée à la baisse du sodium échangeable. Les travaux de Zhao et al. (2018) ont montré une baisse du pH du sol de 0,04 unité soit 32 à 36 fois inférieure aux baisses obtenues.

Ce résultat s'explique par la pureté du gypse utilisé car le gypse issu de la désulfuration des fumées de gaz contient d'autres agents alcalins (Clark et al., 2001). Pour les travaux de Gharaibeh et al. (2009), le pH du sol de la Vallée du Jourdain baisse de 0,28 unité avec l'accroissement de la dose d'apport de gypse de 5 à $20 \mathrm{t} \mathrm{ha}^{-1}$. Cette faible baisse par rapport à nos résultats s'explique par la qualité modérément saline de l'eau d'irrigation utilisée par ces auteurs. En augmentant cependant la dose d'apport du gypse au-delà de $20 \mathrm{t} \mathrm{ha}^{-1}$ jusqu'à $35 \mathrm{t} \mathrm{ha}^{-1}$, ils n'ont pas trouvé d'effet sur le pH du sol. La plus forte baisse du pH du sol obtenue par le mode GrC est en lien avec les travaux de Makoi \& Ndakidemi (2007) qui ont trouvé une baisse du pHKCl de 2,8 unités. Cela confirme l'importante amélioration du $\mathrm{pH}$ des sols sodiques par l'amendement continu du gypse pendant deux ans.

La baisse de la concentration du $\mathrm{Na}^{+}$échangeable du sol et du $\mathrm{pH}$ du sol a pour corollaire, la réduction des processus d'alcalinisation et de sodification. Cela réduit la déstructuration de la couche superficielle du sol qui enduit la perte par lessivage et par ruissellement du carbone et des nutriments du sol. De plus, l'augmentation de la concentration en $\mathrm{Ca}^{2+}$ échangeable du sol est attribuée à celle des ions $\mathrm{Ca}^{2+}$ retenus dans les colloïdes du sol grâce à l'apport du gypse. Cela améliore les liaisons entre les particules d'argile et la matière organique du sol ainsi que la rétention des éléments nutritifs et de l'azote organique. Ainsi, les teneurs en C-total et en N-total du sol qui constituent le pool nutritif augmentent.

Des résultats similaires d'études conduites en une année ont été obtenus par Singh \& Singh (2014) en Inde et par Hanay et al. (2004) en Turquie. L'absence d'effet des modes d'apport du gypse (GrA et $\mathrm{GrC}$ ) par rapport à la PP sur les teneurs en P-total du sol est inattendue. De même, la hausse du P-Olsen observée dans les sols fortement dégradés avec le mode GrC est inattendue. En effet, selon les résultats trouvés par Elrashidi et al. (2010), l'apport du gypse augmente la sorption du P sous forme précipité de phosphate de Ca en raison de la hausse de la concentration en ions $\mathrm{Ca}^{2+} \mathrm{du}$ sol.

La baisse des teneurs en K-total résultant des deux modes de gestion du gypse, corroborent les travaux de Dao (2017) au Burkina Faso, de Schultz et al. (2017) aux Etats-Unis d'Amérique et de Singh \& Singh (2014) en Inde. Cette baisse est plus forte avec le mode GrC comparativement au mode GrA sur chacun des deux niveaux de dégradation du sol. Elle est dépendante de l'activité des ions $\mathrm{Ca}^{2+}$ libérés par le gypse dissout. Contrairement à nos résultats, Elrashidi et al. (2010) conduisant un essai en pots, ont trouvé que l'augmentation de la dose de gypse augmente la teneur en $\mathrm{K}$ du sol mais cette augmentation n'est pas proportionnelle à la quantité de gypse apportée. Ils ont expliqué cette augmentation par le fait que les ions $\mathrm{Ca}^{2+}$ pourraient remplacer les ions $\mathrm{K}^{+}$ sur les sites échangeables ainsi que les ions $\mathrm{K}^{+}$piégés sous une forme non échangeable (couche intermédiaire) dans les minéraux argileux de type 2:1 (montmorillonite, vermiculite et micas). Dans 
nos sols, le type d'argile est la kaolinite, qui est une argile de type 1:1 (Sou, 2009). La différence du type d'argile expliquerait donc en partie la différence avec nos résultats.

L'application des modes d'apport du gypse comparativement à la pratique paysanne (PP) n'améliore pas la CEC effective du sol. Cela pourrait s'expliquer par l'absence d'augmentations importantes des teneurs en matière organique du sol et d'argiles susceptibles d'entraîner une augmentation de la CEC effective. L'augmentation de la somme des bases échangeables est liée à celle du $\mathrm{Ca}^{2+}$ échangeable du sol.

Réponses du sol en fonction de son état de dégradation et du mode d'apport de gypse.

Nos résultats indiquent que la baisse de la concentration en $\mathrm{Na}^{+}$échangeable et du $\mathrm{pH}$ du sol par l'application des modes d'apport du gypse est fonction de l'état de dégradation du sol. Plus le niveau de dégradation du sol est avancé, plus la baisse de la concentration en $\mathrm{Na}^{+}$échangeable du sol et du pH du sol est importante. De plus, la baisse du pH du sol dépend du mode d'apport du gypse. L'apport continu du gypse $(\mathrm{GrC})$ induit la plus forte baisse du $\mathrm{pH}$ dans chacun des deux niveaux de dégradation du sol. Cela serait dû à la concentration élevée en $\mathrm{Ca}^{2+}$ échangeable de la solution du sol avec le mode GrC (Gharaibeh et al., 2014).

Par contre, entre les modes d'apport du gypse, aucune différence sur la concentration en $\mathrm{Na}^{+}$ échangeable du sol n'a été trouvée dans chacun des deux niveaux de dégradation. Ce résultat semble converger aux résultats trouvés par Armstrong \& Tanton (1992). Ces deux auteurs ont conclu en effet que l'apport continu du gypse au sol peut être moins efficace pour réduire le pourcentage en $\mathrm{Na}^{+}$échangeable du sol quoi que les sites d'échange du sol jouent le réservoir qui influe sur le gypse dissout et que les concentrations élevées du $\mathrm{Na}^{+}$échangeable du sol constitue un potentiel de meilleure dissolution du gypse.

Sur les nutriments et le pool nutritif du sol, nos résultats indiquent que l'augmentation des teneurs en $\mathrm{C}$-total et en $\mathrm{N}$-total du sol dépend de son état de dégradation et du mode d'apport de gypse. Sur les sols moyennement dégradés, l'augmentation des teneurs en C-total et en N-total du sol observée avec le mode GrA par rapport à la $\mathrm{PP}$ et à la $\mathrm{GrC}$ illustre la réduction des phénomènes d'alcalinisation et de sodification du sol ainsi que l'amélioration des liaisons entre les argiles et les matières organiques du sol. Quant à la baisse déductive des teneurs en C-total et en N-total par l'apport continu du gypse $(\mathrm{GrC})$ comparativement à la PP et à $\mathrm{GrA}$ sur ces sols moyennement dégradés, elle pourrait être le fait d'une plus grande activité microbienne avec une intense minéralisation en raison de la quantité importante de gypse apportée par ce traitement GrC.

En effet, l'augmentation des ions $\mathrm{Ca}^{2+}$ du sol provenant de la dissolution de quantité importante de gypse amendée sur ces sols augmente la stabilité structurale du sol (Armstrong \& Tanton, 1992; Chen \& Dick, 2011; Dao, 2017) grâce aux liaisons calciques solides qui empêchent la dispersion des argiles et des matières organiques. Il en résulte une meilleure aération et une meilleure rétention d'eau du sol (Bahçecí, 2009; Chaganti et al., 2015; Gharaibeh et al., 2009) qui constituent des facteurs stimulants de l'augmentation de la vie et de l'activité microbienne telluriques (Schultz et al., 2017).

Ces augmentations de la concentration en $\mathrm{Ca}^{2+}$ échangeable du sol constatées par l'application continu de gypse $(\mathrm{GrC})$ comparativement à la $\mathrm{PP}$ dans les sols moyennement dégradés et dans les 
Effets résiduel et d’apport continu de gypse sur les propriétés chimiques d’u...

sols fortement dégradés montrent que la solubilisation du Ca du gypse dépend de la quantité de gypse apportée par ce traitement $\mathrm{GrC}$ et à la concentration en $\mathrm{Na}^{+}$échangeable du sol (Armstrong \& Tanton, 1992). Ce résultat est confirmé par la plus forte augmentation de la somme des bases échangeables observée avec l'application du mode GrC dans les sols fortement dégradés.

\section{Effets des modes d'apport du gypse sur les rendements du maïs}

Les rendements paille et grains ont été améliorés par l'application des modes d'apport du gypse. Ce résultat est en lien avec l'amélioration des propriétés du sol et qui a été rapporté par certains auteurs. Zhao et al. (2018) ont trouvé que l'apport du gypse aux Solonchak en Chine à pH allant de 8,2 et 9,5 accroît graduellement le rendement grains du maïs de $70 \%$ en première année et de $90 \%$ en quatrième année par rapport au traitement sans apport de gypse. Le rendement en grains du maïs évalué après la deuxième année d'apport du gypse par Makoi \& Ndakidemi (2007) sur les sols alluvionnaires sodiques avec un $\mathrm{pH}(\mathrm{KCl})$ de 9,3 a augmenté de 6 fois par rapport à la pratique sans apport de gypse.

Tous ces auteurs ont attribué ces augmentations de rendements aux améliorations observées sur les propriétés physiques et chimiques des sols dues à la libération des ions $\mathrm{Ca}^{2+}$ dans la solution du sol à la suite des amendements gypse et de fumure organique. En effet, la baisse de la concentration en $\mathrm{Na}^{+}$échangeable des sols grâce aux ions $\mathrm{Ca}^{2+}$ induit la réduction du phénomène d'alcalinisation et améliore la stabilité de la structure du sol et sa rétention en eau (Bahçecí, 2009; Chaganti et al., 2015; Gharaibeh et al., 2009). Cela offre alors aux racines des plantes, de meilleures conditions de nutrition. D'autre part, l'accroissement de la fertilité du sol (C-total, N-total et P-Olsen) implique une meilleure disponibilité des nutriments aux plantes du maïs.

En effet, les hausses des exportations en N, P et K et les baisses des exportations de Na par les récoltes de paille et de grains du maïs corroborent l'amélioration des conditions de nutrition du sol par les modes d'apport du gypse. Mühling \& Läuchli (2002) ont confirmé l'augmentation de la concentration en Na dans les feuilles du maïs avec l'augmentation de la concentration en Na de la solution du sol. Cette hausse de la concentration en Na dans la plante de maïs est mise en avant pour expliquer les effets dépressifs sur les rendements à travers les stress osmotiques, ioniques et oxydatifs provoqués.

L'augmentation des rendements paille et grains consécutive à l'application des modes d'apport du gypse aux sols fortement dégradés confirme que la réponse du maïs aux modes d'apport du gypse dépend du niveau de dégradation du sol. Cela s'explique par la forte incidence des effets dépressifs de l'alcalinité et de la sodicité du sol sur les rendements au niveau des sols fortement dégradés (Grattan \& Grieve, 1992) d'une part et d'autre part; par les apports de nutriments liés à la gestion des champs (irrigations et fertilisations) (Sou, 2009). En effet, le gradient de dégradation du sol du site de Kossodo dû aux accumulations de $\mathrm{Na}^{+}$échangeable est contrôlé par l'intensité des irrigations et par les débordements des canaux d'irrigation vers les champs.

Les fortes hausses des exportations de N, de $\mathrm{P}$ et de $\mathrm{K}$ par l'application de gypse comparativement à la pratique paysanne illustrent l'effet dépressif de l'alcalinité et de la sodicité sur les rendements dans les sols fortement dégradés. L'absence d'effet sur les rendements observés par l'application des modes d'apport du gypse comparativement à la PP dans les sols moyennement dégradés se justifient du fait de la faible incidence de l'état modéré de l'alcalinité du sol sur la croissance et 
le développement des plantes (Miura, 2013) ainsi qu'à l'historique de la gestion de la fertilité des parcelles.

L'apport continu en deux ans du gypse $(\mathrm{GrC})$ aux sols moyennement dégradés semble induire une faible efficacité pour l'exportation des nutriments N, P et K par la paille et les grains du maïs.

Aussi, l'application des modes d'apport du gypse par rapport à la pratique paysanne a modifié la régulation des exportations du $\mathrm{Na}$, du Ca et du K par le maïs. Cette modification observée dans les sols moyennement dégradés montre que le maïs réduit l'accumulation du $\mathrm{Na}$ et du Ca de la paille par une augmentation des exportations du K suite à l'application du gypse comparativement à la PP. Ce résultat contraste ceux trouvés par Maas \& Grieve (1987). Ces auteurs ont trouvé que lorsque le ratio des concentrations $\left[\mathrm{Na}^{+}\right] /\left[\mathrm{Ca}^{2+}\right]$ de la solution du sol décroît, les concentrations en ions $\mathrm{Na}^{+}$et $\mathrm{Ca}^{2+}$ dans les plantes de maïs subissent respectivement de fortes baisses et augmentations. Rengel (1992) trouve que dans ces conditions du sol, les concentrations des ions $\mathrm{K}^{+}$des feuilles du maïs augmentent.

\section{Conclusion}

Les terres irriguées sont en proie à la dégradation résultant de processus d'alcalinisation principalement dus à l'utilisation d'eaux polluées. Améliorer la qualité des sols et la productivité des cultures des systèmes irrigués est une préoccupation majeure en Afrique subsaharienne au regard de la contribution de ce secteur rural dans la lutte contre l'insécurité alimentaire, la sousemployabilité et la pauvreté. Ce travail a contribué à déterminer les effets des modes d'apport du gypse sur la remédiation des propriétés chimiques d'un sol maraîcher urbain ainsi que sur la productivité du maïs en fonction de l'état de dégradation du sol.

L'étude montre que les modes d'apport du gypse baissent la concentration en $\mathrm{Na}^{+}$échangeable et le pH du sol. Ces baisses dépendent cependant du niveau de dégradation du sol. Plus la dégradation est forte, plus la baisse de la concentration en $\mathrm{Na}^{+}$échangeable et du $\mathrm{pH}$ du sol est forte. Ce travail suggère que les modes d'apport du gypse augmentent les teneurs en C-total et en N-total sur les sols moyennement dégradés et les teneurs en P-Olsen sur les sols fortement dégradés. Sur les rendements en paille et en grains du maïs, les modes d'apport du gypse ( $\mathrm{GrA}$ et $\mathrm{GrC}$ ) ont induit une augmentation uniquement sur les sols fortement dégradés. Cela implique que sur les sols moyennement dégradés, l'apport continu du gypse n'est pas recommandé.

Ces résultats obtenus confirment les hypothèses selon lesquelles les modes d'apport du gypse améliorent les propriétés chimiques du sol et les performances du maïs. Nous recommandons ainsi aux producteurs, l'application des doses raisonnées de gypse au sol alcalinisé du site maraicher qui constitue une solution de récupération des sols dégradés au profit de l'agriculture. Des solutions devraient être envisagées pour réduire fortement l'alcalinité de l'eau de la STEP. D'autres études devraient étayer l'impact de ces modes d'apport du gypse sur les propriétés physiques et biologiques du sol afin d'appréhender les mécanismes qui soutiennent la remédiation des sols alcalins affectés.

\section{Remerciements}

Les travaux de recherche de cette étude ont été financés par le projet UrbanFoodPlus 
Effets résiduel et d'apport continu de gypse sur les propriétés chimiques d'u...

(www.urbanfoodplus.org) dans le cadre de l'initiative GlobE (BMBF, 031A242A), en tant que fonds de participation du ministère fédéral allemand de l'éducation et de la recherche (BMBF) et du ministère fédéral de la Coopération économique et du Développement (BMZ). Nous remercions les agriculteurs du site maraîcher urbain de Kossodo, les personnels des laboratoires PhysicalGeographical Laboratory of Ruhr- Universität Bochum, Sol-Eau-Plante (SEP) de Kamboinsé et Organic Plant Production and Agroecosystems Research in the Tropics and Subtropics de Universität Kassel, le Dr Christoph STEINER et les Prof. Dr Bernd MARSCHNER et Andreas BUERKERT pour leur assistance.

\section{Bibliographie}

1. Qadir M. 2016. Reversing salt-induced land degradation requires integrated measures. Water Economics and Policy, 2, 1671001-8.

2. Koné D., 2010. Making urban excreta and wastewater management contribute to cities ' economic development: a paradigm shift. Water Policy, 12, 602-610.

3. Dao J., 2017. Effects of irrigation water quality on soil properties and crops in urban gardens of Ouagadougou, Burkina Faso. Doktor der Agrarwissenschaften: Universität Kassel (Germany). $104 \mathrm{pp}$.

4. Sou Y. M., 2009. Recyclage des eaux usées en irrigation: potentiel fertilisant, risques sanitaires et impacts sur la qualité des sols. Docteur ès Science : École Polytechnique Fédérale de Lausanne (Suisse). 162pp.

5. Drechsel P., Graefe S., Sonou M. \& Cofie O. O., 2006. Informal Irrigation in Urban West Africa: An Overview,. Colombo, Sri Lanka.

6. Mahanta K., K., Kansal M. L. \& Mishra G. C., 2015. Managing salt affected soils : Issues and strategies. Journal of Soil Science and Environmental Management, 4, 1-9.

7. Armstrong B. S. A. \& Tanton W. T., 1992. Gypsum applications to aggregated saline-sodic clay topsoils. Journal of Soil Science, 43, 249-260.

8. Chun S., Nishiyama M. \& Matsumoto S., 2001. Sodic soils reclaimed with by-product from flue gas desulfurization: corn production and soil quality. Environmental Pollution, 114, 453-459.

9. Kitila K., Chala A. \& Workina M., 2020. Effect of Gypsum and compost Application in Reclaiming Sodic soils at Small Scale Irrigation Farm in Bora District of East Shewa Zone, Oromia, Ethiopia. Agriways, 8, 28-44.

10. Wong V. N. L., Dalal R. C. \& Greene R. S. B., 2008. Salinity and sodicity effects on respiration and microbial biomass of soil. Biology and Fertility of Soils, 44, 943-953.

11. Carter M. R., 1986. Microbial biomass and mineralizable nitrogen in Solonetzic soils: Influence of Gypsum and Lime amendments. Soil Biol. Biochem., 18, 531-537.

12. Schultz E., Chatterjee A., DeSutter T. \& Franzen D., 2017. Sodic Soil Reclamation Potential of Gypsum and Biochar additions: Influence on Physicochemical Properties and Soil Respiration. Communications in Soil Science and Plant Analysis, 48, 1792-1803. 
13. Abrol I. P., Yadav J. S. P. \& Massoud F. I., 1988. Salt-Affected Soils and their Management. Soils Resources. FAO, Rome, Italy.

14. Zhao Y., Wang S., Li Y., Liu J., Zhuo Y., Zhang W., Wang J. \& Xu L., 2018. Long-term performance of flue gas desulfurization gypsum in a large-scale application in a saline-alkali wasteland in northwest China. Agriculture, Ecosystems and Environment, 261, 115-124.

15. Mao Y., Li X., Dick W. A. \& Chen L., 2016. Remediation of saline - sodic soil with flue gas desulfurization gypsum in a reclaimed tidal flat of southeast China. Journal of environmental sciences, 45, 224-232.

16. Chaganti V. N. \& Crohn D. M., 2015. Evaluating the relative contribution of physiochemical and biological factors in ameliorating a saline-sodic soil amended with composts and biochar and leached with reclaimed water. Geoderma, 259-260, 45-55.

17. Gharaibeh A. M., Eltaif I. N. \& Shunnar F. O., 2009. Leaching and reclamation of calcareous Saline-sodic soil by moderately Saline and moderate-SAR water using Gypsum and Calcium Chloride. Journal of Plant Nutrition and Soil Science, 172, 713-719.

18. Ayers R. S. \& Westcot D. W., 1976. Water quality for agriculture. Soils Resources. FAO, Rome, Italy.

19. Rasouli F., Kiani P. A. \& Karimian N., 2013. Wheat yield and physico-chemical properties of a sodic soil from semi-arid area of Iran as affected by applied gypsum. Geoderma, 193-194, 246255.

20. Elrashidi A. M., West T. L., Seybold A. C., Benham C. E., Schoeneberger J. P. \& Ferguson R., 2010. Effects of Gypsum Addition on Solubility of Nutrients in Soil Amended With Peat. Soil Science, 175, 162-172.

21. Clark B. R., Ritchey D. K. \& Baligar C. V., 2001. Benefits and constraints for use of FGD products on agricultural land. Fuel, 80, 821-828.

22. Zhao Y., Wang S., Li Y., Liu J. \& Zhuo Y., 2018. Extensive reclamation of saline-sodic soils with flue gas desulfurization gypsum on the Songnen Plain, Northeast China. Geoderma, 321, 52-60.

23. WRB I. W. G., 2015. World Reference Base for Soil Resources 2014, update 2015: International soil classification system for naming soils and creating legends for soil maps.In : FAO, eds. World Soil Resources Reports No. 106, Rome.

24. CEDEAO, CILSS, \& UEMOA, 2016. Catalogue Régional des Espèces et Variétés végétales. Abuja-Ouagadougou. 109pp.

25. Naumann C. \& Bassler R., 1976. Methodenbuch: Die chemische Untersuchung von Futtermitteln. Band III. In : Handbuch der Landwirtschaftlichen Versuchs- und Untersuchungsmethodik, 3rd ed. vol. 3. VDLUFA - Verlag, Darmstadt, Germany.

26. Olsen S. R., Cole C. V., Watanabe S. F. \& Dean A. L., 1954. Estimation of available phosphorus in soils by extraction with sodium bicarbonate. Circular, 939, 1-19.

27. Pansu M. \& Gautheyrou J., 2006. Handbook of Soil Analysis: Mineralogical, Organic and 
Effets résiduel et d’apport continu de gypse sur les propriétés chimiques d'u...

Inorganic Methods. Springer, Berlin Heidelberg New York. 996pp.

28. Ciesielski H. \& Sterckeman T., 1997. Determination of cation exchange capacity and exchangeable cations in soils by means of cobalt hexamine trichloride : Effects of experimental conditions. Agronomie, 17, 1-7.

29. R Core Team, 2018. R: a language and environment for statistical computing. R Foundation for Statistical Computing. Vienna, Austria.

30. Bates D., Maechler M., Bolker B. \& Walker S., 2015. Fitting Linear Mixed-Effects Models Using lme4. Journal of Statistical Software, 67, 1-48.

31. Kuznetsova A., Brockhoff P. B. \& Christensen R. H. B., 2017. lmerTest Package : Tests in Linear Mixed Effects Models. Journal of Statistical Software, 82, 1-26.

32. Hothorn T., Bretz F. \& Westfall P., 2008. Simultaneous Inference in General Parametric Models. Biometrical Journal, 50, 346-363.

33. Pinheiro J., Bates D., DebRoy S., Sarkar D. \& R Core Team, 2018._nlme: Linear and Nonlinear Mixed Effects Models. R package version 3.1-137.

34. Qadir M., Schubert S., Ghafoor A. \& Murtaza G., 2001. Amelioration strategies for sodic soils: A review. Land Degradation and Development, 12, 357-386.

35. Rengasamy P., 1987. Importance of Calcium in Irrigation with Saline-Sodic Water - A Viewpoint. Agricultural Water Management, 12, 207-219.

36. Gharaibeh A. M., Rusan J. M., Eltaif I. N. \& Shunnar F. O., 2014. Reclamation of highly calcareous saline-sodic soil using low quality water and phosphogypsum. Applied Water Science, 4, 223-230.

37. Mahmoodabadi M., Yazdanpanah N., Pazira E. \& Neshat A., 2013. Reclamation of calcareous saline sodic soil with different amendments (I): Redistribution of soluble cations within the soil profile. Agricultural Water Management, 120, 30-38.

38. Kim Y. J., Choo B. K. \& Cho J. Y., 2017. Effect of gypsum and rice straw compost application on improvements of soil quality during desalination of reclaimed coastal tideland soils: Ten years of long-term experiments. Catena, 156, 131-138.

39. Clark J. G., Dodgshun N., Sale G. W. P. \& Tang C., 2007. Changes in chemical and biological properties of a sodic clay subsoil with addition of organic amendments. Soil Biology and Biochemistry, 39, 2806-2817.

40. Makoi R. J. H. J. \& Ndakidemi A. P., 2007. Reclamation of sodic soils in northern Tanzania, using locally available organic and inorganic resources. African Journal of Biotechnology, 6, 19261931.

41. Singh A. \& Singh K. J., 2014. Effect of Gypsum on the Reclamation and Soil Chemical Properties in Sodic Soils of Raebareli District, Uttar Pradesh. International Journal of Scientific Research in Environmental Sciences, 2, 429-434. 
42. Hanay A., Büyüksönmez F., Kiziloglu M. F. \& Canbolat Y. M., 2004. Reclamation of Saline-Sodic Soils with Gypsum and MSW Compost. Compost Science \& Utilization, 12, 175-179.

43. Chen L. \& Dick A. W., 2011. Gypsum as an agricultural amendment: General use guidelines. Bulletin, 945, 35.

44. Bahçecİ İ., 2009. Determination of salt leaching and gypsum requirements with field tests of saline-sodic soils in central Turkey. Irrigation and Drainage, 58, 332-345.

45. Chaganti N. V., Crohn M. D. \& Šimůnek J., 2015. Leaching and reclamation of a biochar and compost amended saline-sodic soil with moderate SAR reclaimed water. Agricultural Water Management, 158, 255-265.

46. Mühling H. K. \& Läuchli A., 2002. Effect of salt stress on growth and cation compartmentation in leaves of two plant species differing in salt tolerance. Journal of plant physiology, 159, 137-146.

47. Grattan S. R. \& Grieve C. M., 1992. Mineral element acquisition and growth response of plants grown in saline environments. Agriculture, Ecosystems and Environment, 38, 275-300.

48. Miura K., 2013. Nitrogen and Phosphorus Nutrition Under Salinity Stress. In : Ahmad P. et al., eds Ecophysiology and Responses of Plants under Salt Stress. Springer Science+Business Media. 425-441.

49. Maas V. E. \& Grieve M. C., 1987. Sodium-induced calcium deficiency in salt-stressed corn. Plant, Cell \& Environment, 10, 559-564.

50. Rengel Z., 1992. The role of calcium in salt toxicity. Plant, Cell \& Environment, 15, 625-632.

PDF généré automatiquement le 2021-12-21 23:57:03

Url de l'article : https://popups.uliege.be/2295-8010/index.php?id=1935 